\title{
DENSE SCALES OF FUNCTIONS
}

\author{
C. A. ROGERS
}

(received 8 February 1961, revised 1 May, 1961)

\section{Introduction.}

In a recent paper [1] on scales of functions (which will be called $S F$ for short) P. Erdös C. A. Rogers and S. J. Taylor developed the theory of scales of functions and used the Continuum Hypothesis to establish the existence of scales of functions having certain desirable properties. One of these properties was that of being dense in a certain sense. In the course of some joint work Taylor and I have felt the need for scales which are dense in a rather stronger sense. The object of this note is to indicate how the methods of $S F$ can be used to show that the Continuum Hypothesis implies the existence of scales with the required properties.

2. As in $S F$ we use $\mathscr{C}$ to denote the class of real functions $f(x)$ which are positive and continuous for $x \geqq 0$ and satisfy $f(0)=1$. We use $i$ to denote the function $i(x)=1$, for $0 \leqq x \leqq 1, i(x)=x$, for $x \geqq 1$; and $k$ to denote the function $k(x)=1$, for $x \geqq 0$. The relations ' $\prec$ ' of dominance and ' $\sim$ ' of equivalence are introduced into $\mathscr{C}$ in a natural way as in $S F$. Two functions $f, g$ in $\mathscr{C}$ are comparable if

$$
f \prec g \text { or } f \sim g \text { or } g \prec f \text {; }
$$

and are monotonically comparable if, in addition, the ratio $f(x) / g(x)$ is a monotonic function of $x$ for all sufficiently large values of $x$.

It is convenient to list the following properties, introduced in $S F$, which may hold for a subset $\mathscr{S}$ of $\mathscr{C}$ :

$\left(\mathrm{P}_{1}^{*}\right)$ if $f, g \in \mathscr{S}$, then $f$ and $g$ are monotonically comparable;

$\left(\mathrm{P}_{2}\right)$ the functions $k, i$ are in $\mathscr{S}$;

$\left(\mathrm{P}_{3}\right)$ if $f, g \in \mathscr{S}$ and $\alpha, \beta$ are real numbers, then $f^{\alpha} g^{\beta} \in \mathscr{S}$;

$\left(\mathrm{P}_{4}\right)$ if $f, g \in \mathscr{S}$ and $f \neq g$, then $f$ is not equivalent to $g$;

$\left(\mathrm{P}_{5}\right)$ if $f \in \mathscr{C}$ and $f$ is comparable with each element of $\mathscr{S}$, then $f$ is equivalent to at least one element of $\mathscr{S}$;

$\left(\mathrm{P}_{6}\right)$ if $g \in \mathscr{C}$, there is at least one $f$ in $\mathscr{S}$ such that $g \prec f$;

$\left(\mathrm{P}_{7}\right)$ if $g \in \mathscr{C}$ and $k \prec g$, there is at least one $f$ in $\mathscr{S}$ such that $k \prec f<g$.

As in $S F$ a set $\mathscr{S}$ satisfying $\left(\mathrm{P}_{1}^{*}\right),\left(\mathrm{P}_{2}\right)$ and $\left(\mathrm{P}_{3}\right)$ is called a monotone scale of functions. If such a scale has property $\left(\mathrm{P}_{4}\right)$ it is irreducible; if $\left(\mathrm{P}_{5}\right)$ it is 
maximal; if $\left(\mathrm{P}_{6}\right)$ and $\left(\mathrm{P}_{7}\right)$ it is dense. We introduce two further properties, rather similar in their nature to $\left(\mathrm{P}_{7}\right)$.

$\left(\mathrm{P}_{10}\right)$ If $l_{1}, l_{2}, \cdots, u_{1}, u_{2}, \cdots \in \mathscr{S}$ and $l_{1} \prec l_{2} \prec \cdots \prec l_{n} \cdots \cdots \prec u_{m}$ $\prec \cdots \prec u_{2} \prec u_{1}$, there is an $s$ in $\mathscr{S}$ with

$l_{1}<l_{2}<\cdots<l_{n}<\cdots<s<\cdots<u_{m}<\cdots<u_{2}<u_{1}$.

$\left(\mathrm{P}_{11}\right)$ If $f \in \mathscr{C}$ and $l_{1}, l_{2}, \cdots \in \mathscr{S}$ and $l_{1} \prec l_{2} \prec \cdots \prec l_{n} \prec \cdots \prec f$, there is an $s$ in $\mathscr{S}$ with

$l_{1} \prec l_{2} \prec \cdots \prec l_{n} \prec \cdots \prec s \prec f$.

A scale of functions satisfying the four conditions $\left(\mathrm{P}_{6}\right),\left(\mathrm{P}_{7}\right),\left(\mathrm{P}_{10}\right)$ and $\left(P_{11}\right)$ will be called a strongly dense scale.

Our main object in this note is to prove

THEOREM 2. The continuum hypothesis implies the existence of a monotone scale $\mathscr{S}$, which is irreducible, maximal and strongly dense.

We show without difficulty that any monotone scale with the property $\left(\mathrm{P}_{\mathrm{b}}\right)$ also has the property $\left(\mathrm{P}_{10}\right)$. It is also easy to show that any monotone scale $\mathscr{S}$ with the property $\left(\mathrm{P}_{4}\right)$ also has the property $\left(\mathrm{P}_{11}^{\prime}\right)$ obtained from $\left(\mathrm{P}_{11}\right)$ by replacing the condition ' $s \prec f$ ' by the condition 'it is not true that $f \prec s$ '. However, the full property $\left(\mathrm{P}_{\mathbf{1 1}}\right)$ seems to be essentially stronger than $\left(\mathrm{P}_{11}^{\prime}\right)$, and in order to obtain a scale with this property it seems to be necessary to modify the details of the construction used in $S F$.

3. In this section we prove four lemmas, the first three being closely related to Lemma 5 of $S F$.

LEMMA 1. If $l_{1}, l_{2}, \cdots, u_{1}, u_{2}, \cdots$ are monotonically comparable functions of $\mathscr{C}$, satisfying

$$
l_{1} \prec l_{2} \prec \cdots \prec l_{n} \prec \cdots \cdots \prec u_{m} \prec \cdots \prec u_{2} \prec u_{1},
$$

there is a function $s$ of $\mathscr{C}$, monotonically comparable with each of $l_{1}, l_{2}, \cdots$, $u_{1}, u_{2}, \cdots$, and satisfying

$$
l_{1} \prec l_{2} \prec \cdots \prec l_{n} \prec \cdots \prec s \prec \cdots \prec u_{m} \prec \cdots \prec u_{2} \prec u_{1} .
$$

PRoor. Write $v_{m}=u_{m}$ for $m=1,2, \cdots$ and $q_{n}=l_{n}$ for $n=1,2, \cdots$. Apply the construction of Part (i) of the proof of Lemma 5 of $S F$, omitting the condition $v_{r+1}(x)>r f(x)$ for $x \geqq \alpha_{r+1}$ from (5). Then take $s=g$. It is clear from $S F$ that we have

$$
s=g \prec v_{m}=u_{m},
$$

for $m=1,2, \cdots$. Further, since the ratio $s(x) / l_{n+1}(x)=g(x) / q_{n+1}(x)$ is monotonic increasing for all sufficiently large $x$, it follows that $l_{n} \prec s$ for $n=1,2, \cdots$. Finally, as in $S F, s$ is monotonically comparable with each of $l_{1}, l_{2}, \cdots, u_{1}, u_{2}, \cdots$.

LEMMA 2. If $l_{1}, l_{2}, \cdots, u_{1}, u_{2}, \cdots$ are monotonically comparable functions of $\mathscr{C}$, satisfying 


$$
l_{1} \prec l_{2} \prec \cdots \prec l_{n} \prec \cdots \cdots \prec u_{m} \prec \cdots \prec u_{2} \prec u_{1},
$$

and $h$ is a function of $\mathscr{C}$ satisfying

$$
l_{1} \prec l_{2} \prec \cdots \prec l_{n} \prec \cdots<h,
$$

there is a function $s$ of $\mathscr{C}$ monotonically comparable with each of $l_{1}, l_{2}, \cdots$, $u_{1}, u_{2}, \cdots$ and satisfying

$l_{1} \prec l_{2} \prec \cdots \prec l_{n} \prec \cdots \prec s \prec \cdots \prec u_{m} \prec \cdots \prec u_{2} \prec u_{1}, s \prec h$.

PROOF. Write $f=1 / h, v_{n}=1 / l_{n}, n=1,2, \cdots$,

$$
q_{m}=1 / u_{m}, \quad m=1,2, \cdots .
$$

Apply the construction of part (i) of the proof of Lemma 5 of $S F$. Then take $s=1 / g$. It is easy to verify that $s$ satisfies the required conditions. It should be noted that in this work there is no need to use the condition, in the hypotheses of Lemma 5 of $S F$, that $f$ is comparable with the functions $q_{1}, q_{2}, \cdots$.

Lemma 3. The result of Lemma 2 still holds in the case when one or both of the sequences $l_{1}, l_{2}, \cdots$ and $u_{1}, u_{2}, \cdots$ terminate after a finite number of terms.

Proof. If the sequence $u_{1}, u_{2}, \cdots$ terminates but the sequence $l_{1}, l_{2}, \cdots$ does not, the result follows as in the proof of Lemma 2, the modifications necessary being clear. If the sequence $l_{1}, l_{2}, \cdots$ terminates, say with $l_{n}$, we can apply Lemma 5 of $S F$ to the function $l_{n}$ and the finite or infinite sequence $u_{1}, u_{2}, \cdots$, and obtain a function $g$ of $\mathscr{C}$, which is monotonically comparable with $l_{n}, u_{1}, u_{2}, \cdots$, and which satisties

$$
l_{n} \prec g<\cdots<u_{m} \prec \cdots \prec u_{2} \prec u_{1} .
$$

By Lemma 4 of $S F$ we can then find a function $s$ of $\mathscr{C}$, which is monotonically comparable with $l_{n}$ and $g$, and which satisfies

$$
l_{n} \prec s \prec g, \quad l_{n}<s<h .
$$

This function $s$ then satisfies our requirements.

Before we state our next lemma we recall one of the definitions of $S F$. Suppose $\mathscr{T}$ is a set of functions of $\mathscr{C}$ and that $\mathscr{B}$ is a subset of $\mathscr{T}$; we say that $\mathscr{B}$ is a basis for $\mathscr{T}$, if every element in $\mathscr{T}$ can be written as a finite product of real powers of elements in $\mathscr{B}$.

LEMMA 4. Let $\mathscr{T}$ be a monotone irreducible scale with a countable basis. Suppose that $f \in \mathscr{C}$ and that $l_{1}, l_{2}, \cdots$ are monotonically comparable functions of $\mathscr{C}$, which are monotonically comparable with each function $t$ of $\mathscr{T}$, and which satisfy

$$
l_{1} \prec l_{2} \prec \cdots \prec l_{n} \prec \cdots \prec f \text {. }
$$


Then there is a function $s$ of $\mathscr{C}$, monotonically comparable with $l_{1}, l_{2}, \cdots$, and with each $t$ of $\mathscr{T}$, satisfying

and such that

$$
l_{1} \prec l_{2} \prec \cdots \prec l_{n} \prec \cdots \prec s<t,
$$

for each $t$ of $\mathscr{T}$ with $t<f$.

$$
t \prec s \prec f
$$

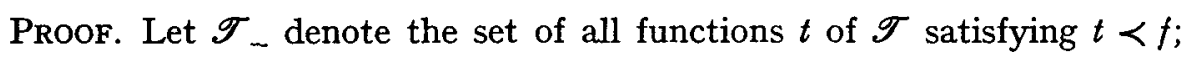
and let $\mathscr{T}_{+}$denote the set of all the other functions of $\mathscr{T}$. The set $\mathscr{T}_{-}$may contain a maximal element $t_{-}$with the property that for all $t$ in $\mathscr{T}$ - other than $t_{-}$we have $t \prec t_{-}$. Otherwise, provided it is not empty, it follows, by the method used in the proof of Lemmas 9 and 10 of $S F$, that we can choose a sequence $t_{1}, t_{2}, \cdots$ of functions of $\mathscr{T}_{-}$, such that

$$
t_{1} \prec t_{2} \prec \cdots,
$$

and such that every function $t$ of $\mathscr{T}_{-}$satisfies $t \prec t_{n}$ for $n$ sufficiently large. In any case, from the functions

$$
l_{1}, l_{2}, \cdots,
$$

if $\mathscr{T}_{-}$is empty, or from the functions

$$
t_{-}, l_{1}, l_{2}, \cdots \text {, }
$$

if $\mathscr{T}_{-}$has a maximal element $t_{-}$, or from the functions

$$
t_{1}, t_{2}, \cdots, l_{1}, l_{2}, \cdots,
$$

in the remaining case, we can choose a sequence $k_{1}, k_{2}, \cdots$ finite or infinite, with $k_{1} \prec k_{2} \prec \cdots$, and such that each function $l_{i}$ satisfies

$$
l_{i} \prec k_{j} \text {, for some } j \text {, }
$$

and each function $t$ of $\mathscr{T}_{-}$satisfies

$$
t \prec k_{j} \text {, for some } j \text {. }
$$

Similarly, if $\mathscr{T}_{+}$is not empty, we can choose a sequence $u_{1}, u_{2}, \cdots$, finite or infinite, from $\mathscr{T}_{+}$such that

$$
\cdots \prec u_{m} \prec \cdots \prec u_{2} \prec u_{1}
$$

and such that each function $t$ of $\mathscr{T}_{+}$satisfies $u_{j} \prec t$ for some $j$. If $\mathscr{T}_{+}$is empty we use Lemma 4 of $S F$ to adjoin a single function to $\mathscr{T}$, in a way which ensures that this element belongs to $\mathscr{T}_{+}$.

By construction the functions $k_{1}, k_{2}, \cdots$ and $u_{1}, u_{2}, \cdots$, are all mutually monotonically comparable, and satisfy

$$
\begin{aligned}
& k_{1} \prec k_{2} \prec \cdots \prec k_{n} \prec \cdots \cdots<u_{m} \prec \cdots \prec u_{2} \prec u_{1}, \\
& k_{1} \prec k_{2} \prec \cdots \prec k_{n} \prec \cdots \prec f .
\end{aligned}
$$


Hence, by Lemmas $\mathbf{2}$ and $\mathbf{3}$, there is a function $s$ monotonically comparable with $k_{1}, k_{2}, \cdots, u_{1}, u_{2}, \cdots$, and satisfying

$$
k_{1} \prec k_{2}<\cdots \prec k_{n} \prec \cdots \prec s \prec \cdots \prec u_{m} \prec \cdots \prec u_{2} \prec u_{1}, s<f .
$$

It is now easy to verify that $s$ has the required properties.

4. Theorem 1. Each monotone maximal scale $\mathscr{S}$ has the property $\left(\mathrm{P}_{10}\right)$.

Proof. Let $l_{1}, l_{2}, \cdots, u_{1}, u_{2}, \cdots$ be functions of $\mathscr{S}$ satisfying

$$
l_{1} \prec l_{2} \prec \cdots \prec l_{n} \prec \cdots \cdots \prec u_{m} \prec \cdots \prec u_{2} \prec u_{1} .
$$

As $\mathscr{S}$ is a monotone scale, the functions $l_{1}, l_{2}, \cdots, u_{1}, u_{2}, \cdots$ are monotonically comparable. So, by Lemma 1 there is a function $g$ of $\mathscr{C}$, monotonically comparable with each of $l_{1}, l_{2}, \cdots, u_{1}, u_{2}, \cdots$, and satisfying

$$
l_{1} \prec l_{2} \prec \cdots \prec l_{n} \prec \cdots \prec g<\cdots<u_{m}<\cdots<u_{2} \prec u_{1} .
$$

By the property $\left(\mathrm{P}_{5}\right)$ there is a function $s$ of $\mathscr{S}$ which satisfies neither $s \prec g$ nor $g \prec s$.

Now $s$ is monotonically comparable with $l_{n}$. If we had $s \sim l_{n}$ or $s<l_{n}$, then $s \prec l_{n+1} \prec g$ contrary to the choice of $s$. Hence $l_{n} \prec s$ for each integer $n$. Similarly $s \prec u_{m}$ for each integer $m$. Thus $\mathscr{S}$ has the property $\left(\mathrm{P}_{10}\right)$ as required.

Proof OF THEOREM 2. It is only necessary to indicate the modifications to be made to the proof of Theorem 5 of $S F$. Initially, instead of well-ordering the set $\mathscr{C}^{\infty}$ of functions of $\mathscr{C}$, which tend to $+\infty$ as $x$ tends to $+\infty$, we well-order the system of sequences $\left\{f_{n}\right\}$, formed from functions of $\mathscr{C}^{\infty}$, as a transfinite sequence

$$
\left\{f_{n}^{(1)}\right\},\left\{f_{n}^{(2)}\right\}, \cdots,\left\{f_{n}^{(\mu)}\right\}, \cdots, \quad \mu<\Omega,
$$

where $\Omega$ is the first ordinal of power greater than $\boldsymbol{\aleph}_{0}$.

Secondly we modify the inductive definition of the sequence of scales $\mathscr{S}_{\mu}$, with $\mu<\Omega$ in two ways. First we replace the function $f_{\mu}$ of $S F$ by the function $f_{0}^{(\mu)}$ and then instead of taking $\mathscr{S}_{\tau}=\mathscr{T}_{\tau}^{\prime \prime \prime}$ we take $\mathscr{S}_{\tau}=\mathscr{T}_{\tau}^{\prime \prime \prime \prime}$, where $\mathscr{T}_{\tau}^{\prime \prime \prime}$ is a new scale defined as follows. If it so happens, that the functions $f_{1}^{(\tau)}, f_{2}^{(\tau)}, \cdots$ are all monotonically comparable with each other and with the functions of $\mathscr{T}_{\tau}^{\prime \prime \prime}$ and they and $f_{0}^{(\tau)}$ satisfy

$$
f_{1}^{(\tau)}<f_{2}^{(\tau)}<\cdots \prec f_{n}^{(\tau)} \prec \cdots \prec f_{0}^{(\tau)},
$$

it follows, by Lemma 4 , that there will be a function $s$ of $\mathscr{C}$ monotonically comparable with each $t$ of $\mathscr{T}_{\tau}^{\prime \prime \prime}$, satisfying

$$
f_{1}^{(\tau)} \prec f_{2}^{(\tau)}<\cdots \prec f_{n}^{(\tau)}<\cdots<s<f_{0}^{(\tau)},
$$

and such that

$$
t<s \prec f_{0}^{(\tau)} \text {, }
$$


for each $t$ of $\mathscr{T}_{\tau}^{\prime \prime \prime}$ with $t<f_{0}^{(\tau)}$. This last condition ensures that $s$ is not equivalent to any function of $\mathscr{T}_{\tau}^{\prime \prime \prime}$; we can consequently form a new irreducible monotone scale $\mathscr{T}_{\tau}^{\prime \prime \prime}$ by taking all the functions of the form $s^{\alpha} t$ where $\alpha$ is real and $t \in \mathscr{T}_{r}^{\prime \prime \prime}$. When the above circumstances do not hold, we simply take $\mathscr{T}_{\tau}^{\prime \prime \prime \prime}=\mathscr{T}_{\tau}^{\prime \prime \prime}$.

As in $S F$ the final scale $S$ has the properties $\left(\mathrm{P}_{1}^{*}\right),\left(\mathrm{P}_{2}\right)-\left(\mathrm{P}_{7}\right)$. By Theorem 1 , it must also have the property $\left(\mathrm{P}_{10}\right)$. Suppose then that $f \in \mathscr{C}$ and $l_{1}, l_{2}, \cdots \in \mathscr{S}$ and

$$
l_{1}<l_{2}<\cdots<l_{n}<\cdots<f .
$$

Then $f / l_{1}, l_{2} / l_{1}, l_{3} / l_{1}, \cdots$ belong to $\mathscr{C}^{\infty}$. So there is an ordinal $\tau$ with $1 \leqq \tau<\Omega$ such that

$$
f_{0}^{(\tau)}=f / l_{1}, f_{r}^{(\tau)}=l_{r+1} / l_{1}, r=1,2, \cdots .
$$

Now the functions $f_{1}^{(\tau)}, f_{2}^{(\tau)}, \cdots$, and the functions of $\mathscr{S}_{7}$ belong to the monotone scale $\mathscr{S}$, so they happen to be monotonically comparable with each other and with the functions of $\mathscr{S}_{\boldsymbol{\tau}}$. Also, they and $f_{0}^{(\boldsymbol{r})}$ satisfy

$$
f_{1}^{(\tau)}<f_{2}^{(\tau)}<\cdots<f_{n}^{(\tau)}<\cdots<f_{0}^{(\tau)} .
$$

Hence the construction of $\mathscr{S}_{\tau}$ ensures the existence of a function $s^{\prime}$ in $\mathscr{S}_{\tau}$ satisfying

$$
f_{\mathbf{1}}^{(\tau)} \prec f_{\mathbf{2}}^{(\tau)}<\cdots \prec f_{n}^{(\tau)} \cdots \prec s^{\prime} \prec f_{0}^{(\tau)} .
$$

So the function $s=s^{\prime} l_{1}$ of $\mathscr{S}$ satisfies

$$
l_{2} \prec l_{3} \prec \cdots \prec s \prec f,
$$

as required. Thus $\mathscr{S}$ has the property $\left(\mathrm{P}_{11}\right)$ and the theorem is proved.

\section{Reference}

[1] Erdos, P., Rogers, C. A. and Taylor, S. J., Scales of functions, Australian Journal of Mathematics 1 (1960) 396.

University College, Gower Street,

London W.C. 1. 